OPEN ACCESS

Edited by:

Vincenzina Fusco,

Institute of Sciences of Food

Production, Italian National Research

Council (CNR), Italy

Reviewed by:

Giuseppe Blaiotta,

University of Naples Federico II, Italy

Rosanna Tofalo,

University of Teramo, Italy

${ }^{*}$ Correspondence:

Shaoquan Liu

chmlsq@nus.edu.sg

Specialty section:

This article was submitted to

Food Microbiology,

a section of the journal

Frontiers in Microbiology

Received: 13 July 2019

Accepted: 20 September 2019

Published: 09 October 2019

Citation:

Gao P, Xia W, Li X and Liu S (2019) Use of Wine and Dairy Yeasts as Single Starter Cultures for Flavor Compound Modification in Fish Sauce

Fermentation

Front. Microbiol. 10:2300 doi: 10.3389/fmicb.2019.02300

\section{Use of Wine and Dairy Yeasts as Single Starter Cultures for Flavor Compound Modification in Fish Sauce Fermentation}

\author{
Pei Gao ${ }^{1,2}$, Wenshui Xia' ${ }^{1}$ Xinzhi $L i^{2}$ and Shaoquan $L_{i u^{2,3 *}}$ \\ 'State Key Laboratory of Food Science and Technology, School of Food Science and Technology, Jiangnan University, Wuxi, \\ China, ${ }^{2}$ Department of Food Science and Technology, National University of Singapore, Singapore, Singapore, ${ }^{3}$ Laboratory \\ of Advanced Food Technology \& 3D Printing, National University of Singapore (Suzhou) Research Institute, Suzhou, China
}

Effects of wine and dairy yeast fermentation on chemical constituents of tilapia fish head hydrolysate supplemented with glucose in an unsalted and acidic environment were investigated. Three wine yeasts (Torulaspora delbrueckii Biodiva, Saccharomyces cerevisiae Lalvin EC-1118 and Pichia kluyveri Frootzen) and one dairy yeast (Kluyveromyces marxianus NCYC1425) were employed as single starter cultures, respectively, and were compared with one soy sauce yeast (Candida versatilis NCYC1433). Each yeast showed different growth kinetics and fermentation performance. Compared with C. versatilis NCYC1433, other yeasts presented a significant higher rate of glucose consumption $(P<0.05)$. Besides, $K$. marxianus NCYC1425 and P. kluyveri Frootzen produced more citric acid and succinic acid, respectively, while S. cerevisiae Lalvin EC-1118 exhibited higher pyruvic acid production. Significant lower levels of total free amino acids were observed in samples inoculated with wine yeasts relative to other yeasts $(P<0.05)$. Non-soy sauce yeasts produced increased various levels of esters and alcohols without traditional fish sauce unpleasant odorants, especially K. marxianus NCYC1425 and P. Kluyveri Frootzen. The results confirmed that non-soy sauce yeasts are suitable for fish sauce flavor compound modification and to develop a fast fermentation process for saltless fish sauce from fish head, which could increase the acceptability of fish sauce and improve the utilization of fish by-products.

\section{Keywords: fish sauce, hydrolysate, tilapia, yeasts, flavor}

\section{INTRODUCTION}

Fish are an extremely important source of protein. In 2015, fish accounted for about 17 percent of global human animal protein consumption, and global fish production peaked at about 171 million tonnes in 2016 (FAO, 2018). However, over 50 percent (w/w) of the weight of fish as by-products are generated accompanying the processing chain (Kristinsson and Rasco, 2000), including heads, tails, bones, skins, scales and intestines. At present, the industrial fish by-products are normally used for the manufacture of low-value products such as animal feed, or even directly discarded as wastes (Kristinsson and Rasco, 2000). These treatments are not only a huge waste of protein resources, but also a pollution of the environment if not treated before disposal. These problems greatly limit the development and utilization of fish by-products. 
Fish sauce is a type of fermented liquid condiments usually made from low-value fish in Southeast and East Asian countries (Lopetcharat et al., 2001). Upon widespread recognition of its ability to impart a savory (umami) taste to dishes, fish sauce has become more accepted globally. A series of physical, biological and chemical reactions occur during the fermentation of fish sauce, including oxidative rancidity of unsaturated fatty acids and the action of exogenous enzymes and endogenous microorganisms (Lopetcharat et al., 2001). As a result, fish sauce is prone to producing undesirable flavor substances such as medium-chain aldehydes, branched-chain carboxylic acids, volatile nitrogen and sulfur-containing compounds (Fukami et al., 2002, 2004; Jiang et al., 2008; Giri et al., 2010; Yoshikawa et al., 2010). These compounds impart distinct fishy, sweaty, rancid and fecal notes to fish sauce, which seriously limit its acceptability. Therefore, there is a pressing need to modify fish sauce flavor so as to attract more consumers.

The usage of microbial inoculation to enhance flavor has become a common practice in fermentation. Zygosaccharomyces rouxii, Candida versatilis or Candida etchellsii and Pichia guilliermondii, isolated from traditional soy sauce, have been effectively employed as starter cultures to enhance the flavor of soy sauce individually or in co-culture with lactic acid bacteria (LAB) (Harada et al., 2017; Singracha et al., 2017; Devanthi et al., 2018). Yoshikawa et al. (2010) reported that inoculation of $Z$. rouxii and $C$. versatilis NCYC1433 coupled with koji and Tetragenococcus halophilus was effective for conferring the soysauce-like flavor and increasing free amino acids and ethanol contents in Chum salmon sauce. To a certain extent, the application of yeasts could reduce the salt level due to production of inhibitory substances such as alcohols (Singracha et al., 2017). But a considerable salt concentration is still essential for the survival of these soy sauce yeasts, which poses health risks to consumers because of high salt levels. Thus, the use of nonhalophilic yeasts to reduce or even eliminate salt addition is of paramount importance. This is the first report on employment of non-soy sauce yeasts for the fermentation of a novel fish sauce from fish head hydrolysate.

In this study, three species of wine yeasts (Torulaspora delbrueckii, Saccharomyces cerevisiae and Pichia kluyveri) and one species of dairy yeast (Kluyveromyces marxianus) with a high aromatic capacity were employed as single starter cultures for fish sauce fermentation, in comparison to one species of soy sauce yeast (C. versatilis). The aim was to ascertain whether nonsoy sauce yeasts were suitable for fish sauce flavor modification and to develop a fast fermentation process for saltless fish sauce with better flavor.

\section{MATERIALS AND METHODS}

\section{Materials}

Nile tilapia (Oreochromis niloticus) heads used in this experiment were prepared in the laboratory from tilapia whole fish that was purchased from a local market (Singapore). Flavourzyme (a fungal protease/peptidase complex) from Aspergillus oryzae ( $\geq 500 \mathrm{U} / \mathrm{g}$ ), was supplied by Sigma-Aldrich Pte Ltd (Saint
Louis, MO, United States). T. delbrueckii Biodiva and S. cerevisiae subsp. bayanus Lalvin EC-1118 were purchased from Lallemand (Brooklyn Park, Australia). P. kluyveri FrootZen was bought from Chr. Hansen (Hørsholm, Denmark). K. marxianus NCYC 1425 and C. versatilis NCYC 1433 were obtained from National Collection of Yeast Cultures (Norwich, England). D-Glucose standard and amino acid standards were purchased from Sigma (Saint Louis, MO, United States). Other chemicals used in this study were of analytical grade.

\section{Preparation of Yeast Starter Cultures}

Prior to inoculation, five yeast strains were respectively transferred from the content of a $25 \%$ glycerine-water solution to YM broth, containing $0.3 \%(\mathrm{w} / \mathrm{v})$ yeast extract, $0.3 \%(\mathrm{w} / \mathrm{v})$ malt extract, $0.5 \%(\mathrm{w} / \mathrm{v})$ peptone and $1 \%(\mathrm{w} / \mathrm{v})$ glucose. After cultivating at $30^{\circ} \mathrm{C}$ for $24 \mathrm{~h}$, yeast cell pellets were harvested by centrifugation (Centrifuge 5810R, Hamburg, Germany) at $12,000 \mathrm{rpm}$ for $10 \mathrm{~min}$ at $4^{\circ} \mathrm{C}$. Then these cell pellets were washed twice with an equivalent volume of $0.85 \%$ saline $(\mathrm{w} / \mathrm{v})$, and re-suspended therein as the starter cultures.

\section{Production of Fish Hydrolysate and Sauce}

The process for the production of fish hydrolysate and sauce is shown in Figure 1. Fish heads were removed from the freezer $\left(-20^{\circ} \mathrm{C}\right)$ to thaw and crushed with a $400 \mathrm{~W}$ Philips HR-1396 Viva Collection Chopper (Amsterdam, Holland). Distilled water was added into the minced fish head ( $\mathrm{w}: \mathrm{v}=1: 6)$, and the $\mathrm{pH}$ of the mixture was adjusted to 3.0 using $90 \%$ lactic acid. This was followed by filtering through a gauze to remove insoluble fish bones and skin. Then the filtrate was placed in a $4^{\circ} \mathrm{C}$ fridge overnight to remove the fat floating on top of the filtrate. The filtrate was subsequently heated in an $85^{\circ} \mathrm{C}$-water bath for $15 \mathrm{~min}$ to deactivate the fish endogenous enzymes.

Thereafter, $\mathrm{pH}$ of the filtrate was shifted to 6.0 by adding 1 $\mathrm{M} \mathrm{NaOH}$, and kept in an $50^{\circ} \mathrm{C}$-oscillating bath (Julabo SW22, Seelbach, Germany) added with $0.5 \%(\mathrm{v} / \mathrm{v})$ protease at a shaking speed of $120 \mathrm{rpm}$ (degree of hydrolysis reached 35-40\%). After $3 \mathrm{~h}$, the hydrolysate was heated at $85^{\circ} \mathrm{C}$ for $15 \mathrm{~min}$ to deactivate the protease. Next, the hydrolysate was added with $2 \%(\mathrm{w} / \mathrm{v})$ glucose and the $\mathrm{pH}$ was shifted to $4.30-4.40$ by adding $90 \%$ lactic acid, and sterilized in a Hirayama HV-50 Upright Autoclave (Saitama, Japan) at $85^{\circ} \mathrm{C}$ (Lopetcharat et al., 2001) for $15 \mathrm{~min}$. Heat treatment was effective for microbes elimination, verified by plating on potato dextrose agar (PDA).

Finally, the cooled sterilized hydrolysate was divided into five portions and each portion was inoculated with the above yeast starter cultures respectively, including S1 (K. marxianus NCYC1425), S2 (T. delbrueckii Biodiva), S3 (S. cerevisiae Lalvin EC-1118), S4 (P. kluyveri Frootzen), and S5 (C. versatilis NCYC1433). These portions were incubated statically at $30^{\circ} \mathrm{C}$ for 7 days, and fermentation was conducted in triplicate for each yeast.

Samples were collected at day $0,1,3,5$ and 7 , and analyzed for yeast enumeration, $\mathrm{pH}$ and chemical constituents. The $\mathrm{pH}$ was measured directly with a $\mathrm{pH}$ meter (Metrohm, 


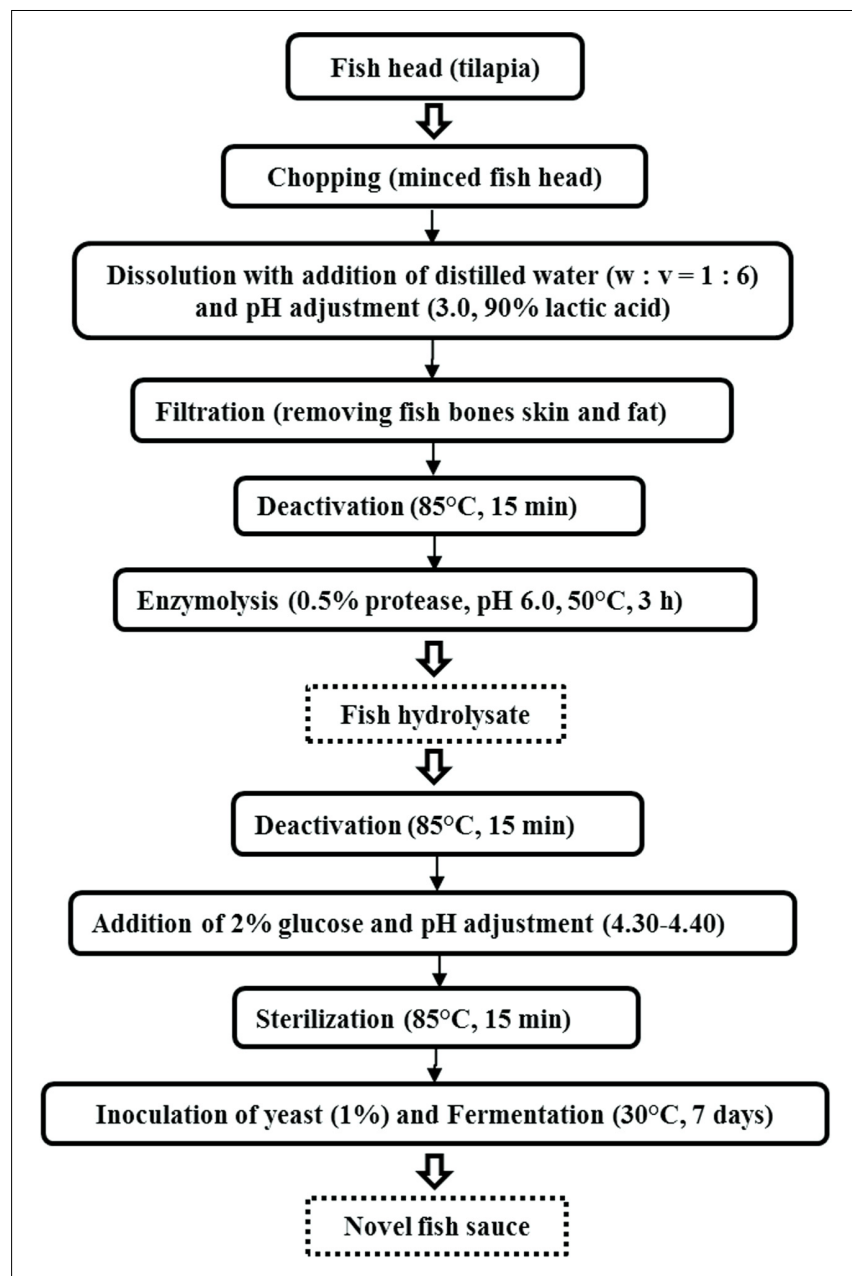

FIGURE 1 | The process for the production of fish hydrolysate and sauce.

Herisau, Switzerland). Yeasts were enumerated using the method of spread plating on PDA medium at $30^{\circ} \mathrm{C}$ for $48 \mathrm{~h}$. The results were expressed as $\log \mathrm{CFU} / \mathrm{mL}$. Each sample was evaluated in triplicate.

\section{Non-volatile Constituents Analysis}

Sugar (glucose) and seven organic acids (citric acid, pyruvic acid, malic acid, succinic acid, lactic acid, and acetic acid) were analyzed according to the method of $\mathrm{Lu}$ et al. (2018) using a Shimadzu HPLC. The mobile phase was acetonitrile and water (v: $\mathrm{v}=80: 20)$, and $0.1 \%(\mathrm{v} / \mathrm{v})$ sulfuric acid, respectively. The results were expressed as $\mathrm{g} / \mathrm{L}$. Each sample was evaluated in triplicate.

Analysis of free amino acids was carried out using the ARACUS Amino Acid Analyzer (MembraPure GmbH, German), connected to a column composed of lithium system $(125 \mathrm{~mm}$ $\mathrm{BL} \times 4.0 \mathrm{~mm}$ ID) and a ammonium absorber $(40 \mathrm{~mm} \times 2.0 \mathrm{~mm}$ ID). Eluent $A$ to Eluent $F$ were used at the flow rate of $240 \mu \mathrm{L} / \mathrm{min}$. The column temperature and the reactor temperature was 50 and $130^{\circ} \mathrm{C}$, respectively. Quantification was performed by using a $100 \mathrm{nmol} / \mathrm{mL}$ external standard solution including 18 amino acids or derivatives (except Cys,
$50 \mathrm{nmol} / \mathrm{mL}$ ). The results were expressed as $\mathrm{mg} / \mathrm{L}$. Each sample was evaluated in triplicate.

\section{Volatile Constituents Analysis}

Volatile compounds of fish sauce were extracted by using the method of head space solid-phase microextraction (HS-SPME) as reported previously (Gao et al., 2016a) with some modification. Each sample was heat treated at $60^{\circ} \mathrm{C}$ for 5 min before extraction. A CAR/PDMS fiber (85 $\mu \mathrm{m}$, Supelco, Bellefonte, $\mathrm{PA}$, United States) was then inserted into the headspace of the sample vial.

Agilent 7890A GC with Agilent 5795C inert MSD with TripleAxis Detector was used for GC-MS analysis. It was carried out according to the previous method (Gao et al., 2016a). The volatile flavor compounds were identified according to NIST 2014 standard library and Kovats index (KI), and 2-octanol $(164.4 \mu \mathrm{g} / \mathrm{L})$ was used as an internal standard. The concentration of volatile compounds was calculated by comparing the peak area of each compound with that of the internal standard, expressed as $\mathrm{mg} / \mathrm{L}$. Each sample was evaluated in triplicate.

\section{Statistical Analysis}

The experiments were designed and analyzed statistically by ANOVA, and mean value differences were evaluated by using Duncan's multiple range test $(P<0.05)$. Statistical analysis was performed using the SPSS statistic program (Version 19.0, SPSS Inc., Chicago, IL).

\section{RESULTS}

\section{Yeast Populations and $\mathrm{pH}$}

In order to accelerate the growth of yeasts, $2 \%(\mathrm{w} / \mathrm{v})$ glucose was added to each inoculated sample. Changes of yeast cell populations in inoculated samples during fermentation are shown in Figure 2A. Yeast populations in all samples presented a first increasing and then decreasing trend. An increase of around $2 \log \mathrm{CFU} / \mathrm{mL}$ was observed in each inoculated sample. In S1S4 (wine and dairy yeasts), the yeast counts reached the level of about $7.5 \log \mathrm{CFU} / \mathrm{mL}$ after day 1 of fermentation, while on day 3 S5 (soy sauce yeast) reached $7.0 \log \mathrm{CFU} / \mathrm{mL}$.

In order to inhibit the growth of pathogenic and spoilage bacteria, the environment of the hydrolysate was adjusted to acidic conditions before fermentation in the absence of LAB. The $\mathrm{pH}$ changes of all samples during fermentation are shown in Figure 2B. At the beginning, the $\mathrm{pH}$ of all samples ranged from 4.3 to 4.4. A slight decreasing trend was observed in all samples during fermentation as a whole until day 5 , when $\mathrm{pH}$ began to rise slightly.

\section{Sugar and Organic Acids}

Sugar (glucose) changes in all samples with yeast treatment are shown in Figure 3A. A significant $(P<0.05)$ decline was observed on day 1 of fermentation in each sample, during which yeast counts of S1-S4 reached the maximum of $7.5 \log \mathrm{CFU} / \mathrm{mL}$ (Figure 2A). No glucose was detected in S1-S4 from day 3, 


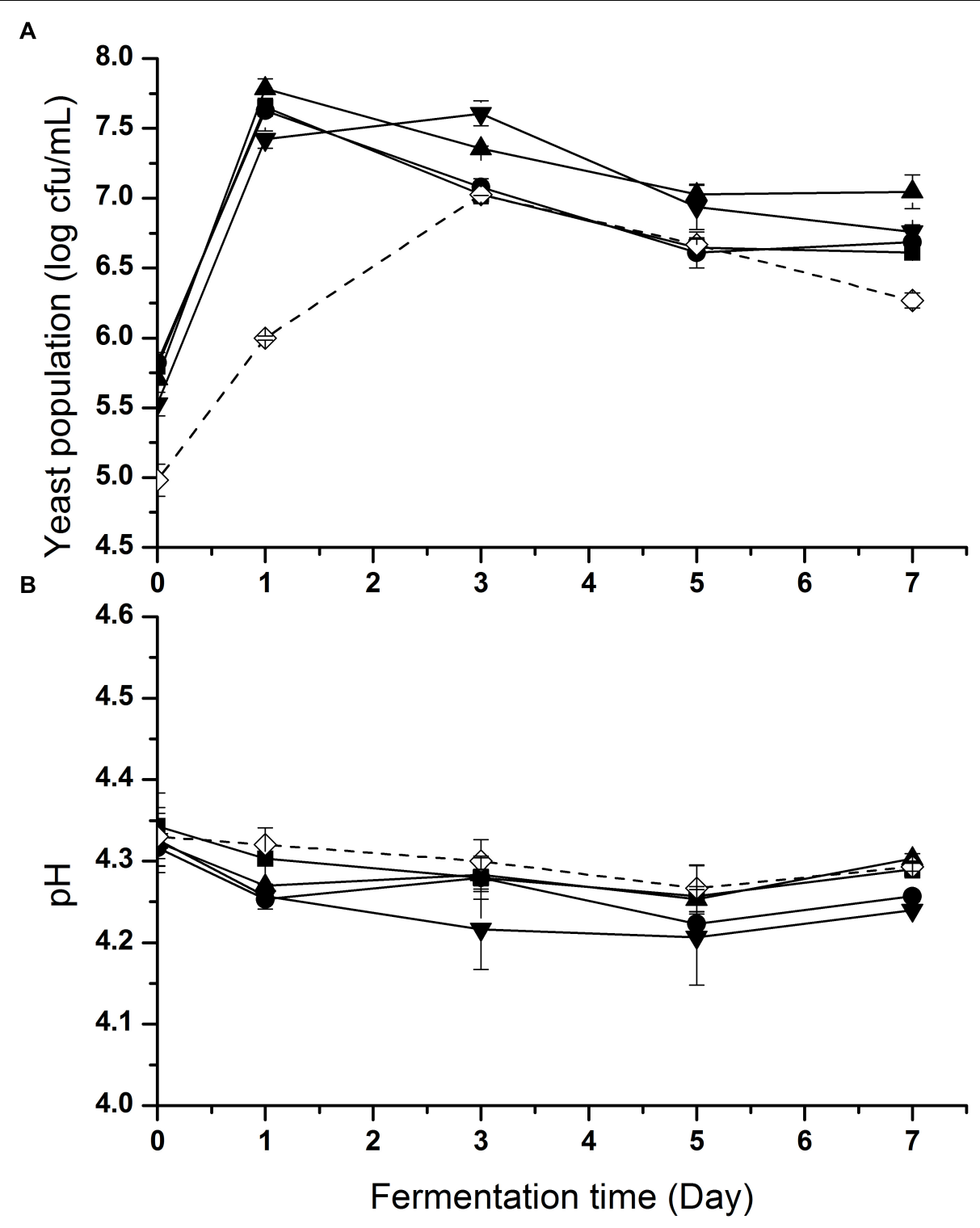

FIGURE 2 | Yeast population (A) and $\mathrm{pH}$ (B) changes of fish sauce during fermentation with different yeasts. S1

inoculated with Kluyveromyces marxianus

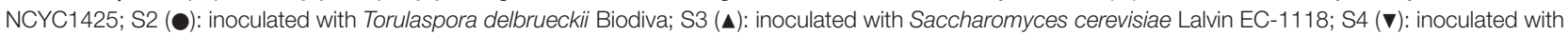
Pichia kluyveri Frootzen; S5 $(\diamond)$ : inoculated with Candida versatilis NCYC1433.

while glucose decreased continuously with a residual content of $3.21 \mathrm{~g} / \mathrm{L}$ on day 7 in S5.

It is well known that organic acids are involved in yeast fermentation. As can be seen, no significant differences were observed in the concentration of lactic acid (Figure 3B) while that of other organic acids significantly increased after the fermentation of yeasts, leading to a slight decrease of $\mathrm{pH}$. The difference was ascribed to sugar metabolism by these yeasts to form "other organic acids" but no or little lactic acid, which is usually produced by LAB through lactic acid fermentation (Lu et al., 2017a). In addition, due to the usage of lactic acid for the acidity adjustment, lactic acid exhibited the highest concentration at the beginning.
Although $P$. kluyveri Frootzen had a lower rate of glucose consumption (Figure 3A), more pyruvic acid (Figure 3C) was produced by $\mathrm{S} 4$ (P. kluyveri Frootzen) within the first 3 days, than others including S3 (S. cerevisiae). Citric acid (Figure 3D), malic acid (Figure 3E) and succinic acid (Figure 3F) are known to be the critical intermediate products of the TCA cycle. The three acids significantly increased at the early stage of fermentation. At the later stage, malic acid remained stable and citric acid slightly reduced, while succinic acid kept increasing with a faster rate in S3 (S. cerevisiae). A significantly $(P<0.05)$ higher level of acetic acid (Figure 3G) was observed in samples inoculated with K. marxianus NCYC1425 (S1) and C. versatilis NCYC1433 (S5), indicating more acetaldehyde was metabolized in S1 and S5 to 

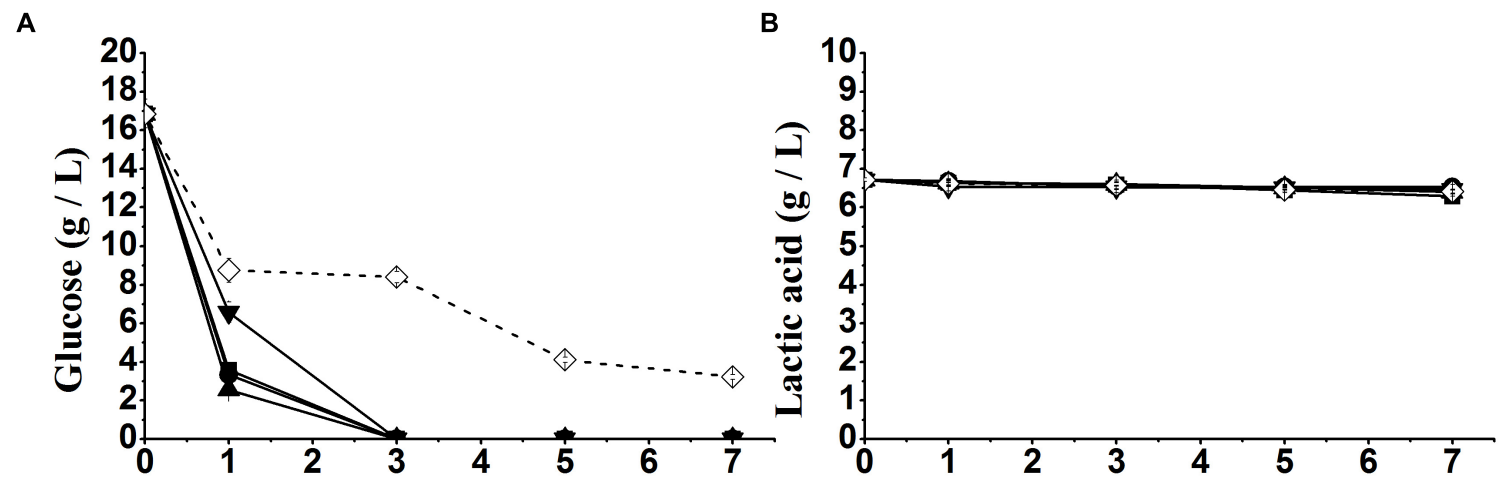

C

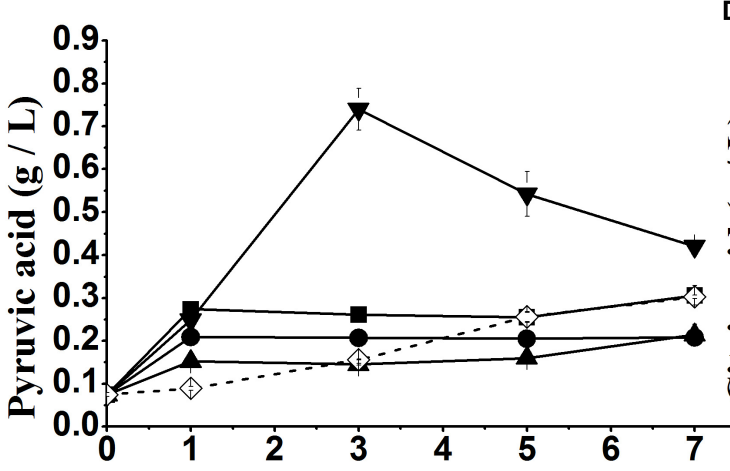

E

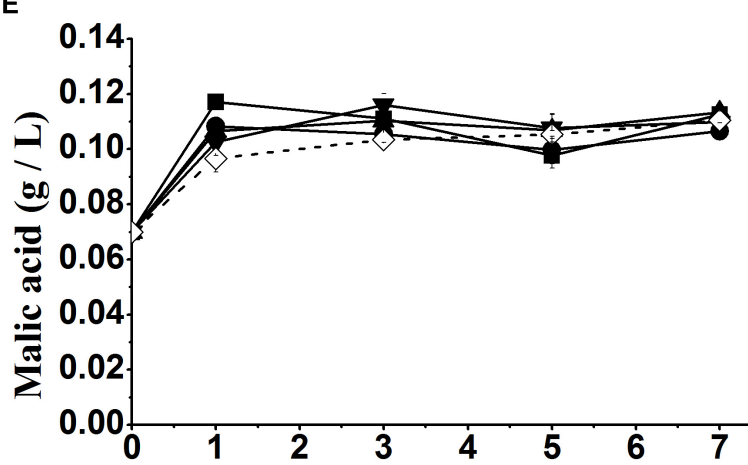

G

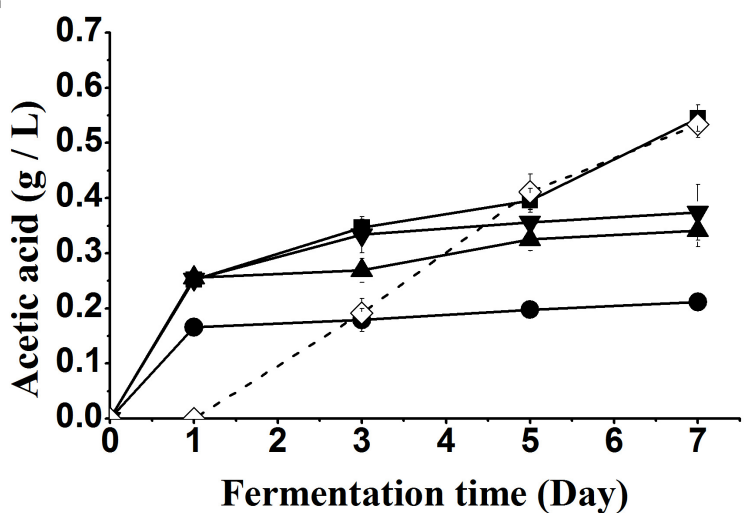

D

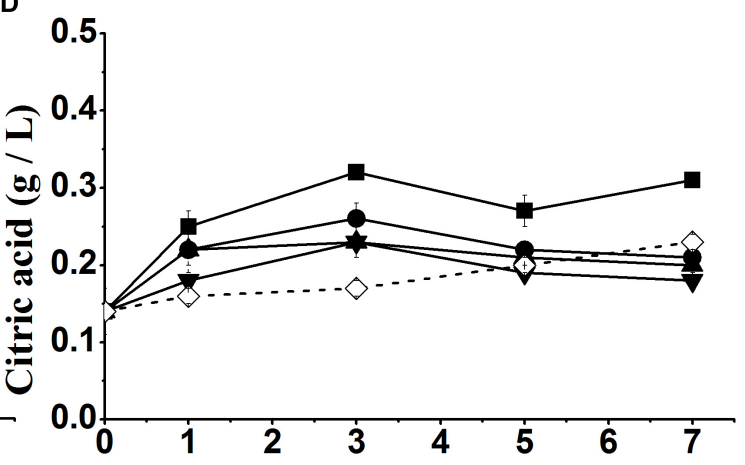

$\mathbf{F}$

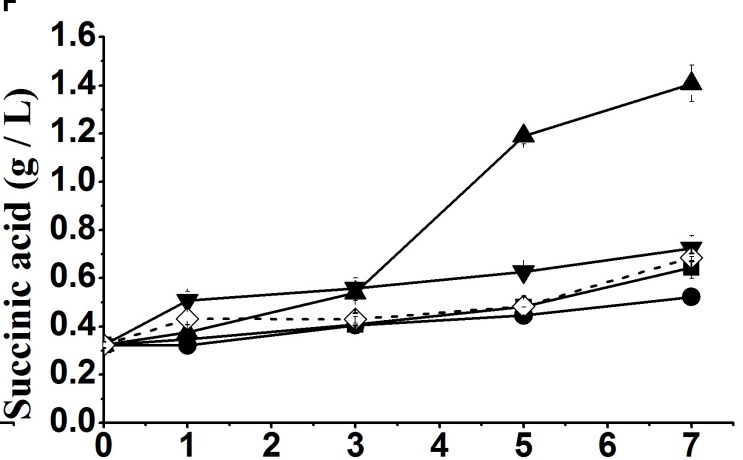

Fermentation time (Day)

FIGURE 3 | Glucose (A) and organic acids (B-F) changes of fish sauce during fermentation with different yeasts. S1 (घ): inoculated with Kluyveromyces marxianus

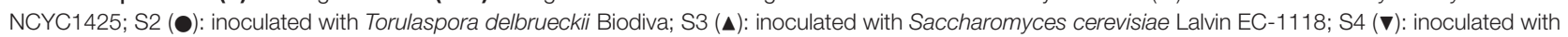
Pichia kluyveri Frootzen; S5 $(\diamond)$ : inoculated with Candida versatilis NCYC1433. 
generate acetic acid. This was also consistent with the result that non-Saccharomyces yeasts were usually high producers of acetic acid reported by Contreras et al. (2014).

\section{Free Amino Acids}

The concentration and composition of free amino acids (FAA) in fish hydrolysate and fish sauce are shown in Table 1. After the flavourzyme treatment, 17 FAA were detected in unfermented fish hydrolysate with concentration of $5296 \mathrm{mg} / \mathrm{L}$. A extremely low concentration $(4 \mathrm{mg} / \mathrm{L})$ was observed in cysteine. A similar result was reported by Robert et al. (2015) who found the lowest amount of cysteine (FFA, 0.04\%) in the by-products (head, frames and viscera) hydrolysate from the same species of Tilapia.

The total FAA of S4 and S5 $(5804 \mathrm{mg} / \mathrm{L}$ and $5966 \mathrm{mg} / \mathrm{L}$, respectively) was significantly $(P<0.05)$ increased compared with fish hydrolysate, indicating that $P$. kluyveri Frootzen and C. versatilis NCYC1433 had a relatively stronger ability of proteolysis to form amino acids. Conversely, the significant $(P<0.05)$ decline of total FAA in S2 (T. delbrueckii, $4657 \mathrm{mg} / \mathrm{L})$ indicated a higher capacity of T. delbrueckii Biodiva to metabolize amino acids. Moreover, all samples except S2 presented more sweet amino acids, among which S1 and S3 presented less bitter amino acids at the same time. The concentration of umami amino acids significantly $(P<0.05)$ decreased in S3, while no significant differences were observed in the other inoculated samples relative to the fish hydrolysate $(P>0.05)$. Individually, significantly lower amounts of threonine, proline, isoleucine and leucine in $\mathrm{S} 1$, threonine and proline in S2, threonine, serine, isoleucine and leucine in S3, isoleucine in S4 were observed compared with S5 $(P<0.05)$. Inversely, $\mathrm{S} 4$ displayed a higher level of alanine than S5 $(P<0.05)$. In addition, a slight decrease in methionine was found in inoculated samples compared with the starting matrix.

\section{Volatile Compounds}

The fish hydrolysate and fish sauce fermented with different yeasts were analyzed for the volatile compounds (Table 2). The main odor components of fish hydrolysate were aldehydes and furans, accounting for $59 \%$ and $22 \%$ of total content respectively. Compared with 34 volatiles before fermentation, 47, 47, 46, 38, and 32 volatiles were detected in S1-S4 (wine and dairy yeasts) and S5 (soy sauce yeast) at the end of fermentation, respectively. And individual concentration of S1-S5 was 2512, 1323, 1590, 8962 , and $848 \mu \mathrm{g} / \mathrm{L}$, respectively, compared to $1142 \mu \mathrm{g} / \mathrm{L}$ of fish hydrolysate. The result indicated that wine yeast and dairy yeast could improve the flavor of fish sauce quantitatively compared with soy sauce yeast, of which P. kluyveri Frootzen displayed the maximum improvement.

Esters were the major volatile compounds produced during the fermentation, mainly consisting of acetate esters and ethyl esters. S4 presented the highest level of esters (P. kluyveri Frootzen, $6621 \mu \mathrm{g} / \mathrm{L}$ ), followed by S1 (K. marxianus, $1120 \mu \mathrm{g} / \mathrm{L}$ ), which were markedly higher than that of S5 $(24 \mu \mathrm{g} / \mathrm{L})$

TABLE 1 | Free amino acids (FAA) in fish sauce fermented with different yeasts (mg/L).

\begin{tabular}{|c|c|c|c|c|c|c|c|}
\hline \multirow[t]{2}{*}{ FAA } & \multirow[t]{2}{*}{ Taste } & \multirow[t]{2}{*}{ Fish hydrolysate } & \multicolumn{5}{|c|}{ Fish sauce (Day 7) } \\
\hline & & & S1 & S2 & S3 & S4 & S5 \\
\hline Asp & Umami & $228.93 \pm 10.66 \mathrm{ab}$ & $228.00 \pm 6.17 \mathrm{ab}$ & $271.30 \pm 65.84 a$ & $204.00 \pm 14.79 b$ & $249.92 \pm 8.50 a b$ & $218.86 \pm 2.95 a b$ \\
\hline Thr & Sweet & $196.15 \pm 14.42 a b$ & $164.94 \pm 2.71 b$ & $168.00 \pm 40.91 b$ & $100.34 \pm 4.18 c$ & $209.85 \pm 5.02 a$ & $212.39 \pm 1.26 \mathrm{a}$ \\
\hline Ser & Sweet & $158.23 \pm 18.08 a$ & $144.88 \pm 8.30 a b$ & $147.55 \pm 20.10 a b$ & $115.92 \pm 20.91 b$ & $172.31 \pm 7.07 \mathrm{a}$ & $170.53 \pm 3.86 a$ \\
\hline Glu & Umami & $526.28 \pm 37.23 a b c$ & $487.69 \pm 28.53 \mathrm{~cd}$ & $544.82 \pm 11.72 a b$ & $466.11 \pm 21.83 d$ & $544.97 \pm 3.52 a$ & $498.82 \pm 16.85 b c d$ \\
\hline Pro & Sweet & $1089.59 \pm 12.20 c$ & $1330.52 \pm 221.36 b$ & $481.47 \pm 7.14 d$ & $1587.64 \pm 9.21 a$ & $1563.47 \pm 13.82 a$ & $1661.33 \pm 24.18 a$ \\
\hline Gly & Sweet & $131.27 \pm 7.45 a$ & $140.08 \pm 5.76 a$ & $142.73 \pm 13.32 a$ & $135.23 \pm 1.93 a$ & $142.78 \pm 2.84 a$ & $135.43 \pm 4.89 a$ \\
\hline Ala & Sweet & $227.48 \pm 8.64 c$ & $268.07 \pm 15.25 b c$ & $278.02 \pm 7.62 b$ & $258.60 \pm 26.42 b c$ & $338.87 \pm 21.81 \mathrm{a}$ & $258.63 \pm 26.05 b c$ \\
\hline Cys & - & $4.19 \pm 0.39 a$ & $3.77 \pm 0.22 \mathrm{ab}$ & $2.59 \pm 0.24 c$ & $3.10 \pm 0.30 \mathrm{bc}$ & $3.39 \pm 0.62 b$ & $3.40 \pm 0.33 b$ \\
\hline Val & - & $307.87 \pm 4.80 \mathrm{a}$ & $281.59 \pm 2.22 b$ & $307.36 \pm 14.44 a$ & $284.36 \pm 15.08 b$ & $282.29 \pm 12.97 b$ & $291.59 \pm 14.58 a b$ \\
\hline Met & Bitter & $169.30 \pm 6.55 a$ & $137.67 \pm 2.12 \mathrm{c}$ & $145.43 \pm 7.21 \mathrm{bc}$ & $139.81 \pm 18.24 c$ & $158.81 \pm 7.35 \mathrm{ab}$ & $162.14 \pm 7.30 a b$ \\
\hline lle & Bitter & $222.73 \pm 8.01 a$ & $181.58 \pm 5.85 c$ & $211.84 \pm 5.12 \mathrm{ab}$ & $177.34 \pm 14.37 \mathrm{c}$ & $193.30 \pm 21.02 b c$ & $226.40 \pm 3.03 a$ \\
\hline Leu & Bitter & $504.61 \pm 19.55 a$ & $414.10 \pm 8.16 \mathrm{~cd}$ & $460.49 \pm 8.20 \mathrm{abc}$ & $372.61 \pm 26.31 d$ & $425.52 \pm 51.87 \mathrm{bc}$ & $471.47 \pm 20.33 a b$ \\
\hline Tyr & Bitter & $275.95 \pm 10.51 \mathrm{ab}$ & $259.58 \pm 7.10 b$ & $269.13 \pm 15.07 a b$ & $269.31 \pm 14.49 a b$ & $254.15 \pm 18.53 b$ & $305.61 \pm 29.92 a$ \\
\hline Phe & Bitter & $399.21 \pm 19.16 \mathrm{bc}$ & $372.67 \pm 14.91 c$ & $410.99 \pm 25.65 b$ & $384.12 \pm 30.51 \mathrm{bc}$ & $384.51 \pm 6.34 \mathrm{bc}$ & $460.22 \pm 13.20 a$ \\
\hline His & Bitter & $118.59 \pm 5.72 a$ & $106.72 \pm 2.22 \mathrm{ab}$ & $113.68 \pm 5.40 a b$ & $104.33 \pm 11.00 b$ & $117.39 \pm 3.63 a$ & $116.55 \pm 2.76 a$ \\
\hline Lys & Bitter & $406.65 \pm 38.16 a b$ & $423.41 \pm 16.72 b$ & $390.52 \pm 29.75 b$ & $382.34 \pm 8.47 b$ & $451.82 \pm 9.37 a$ & $450.22 \pm 3.72 a$ \\
\hline Arg & Bitter & $328.95 \pm 15.87 a$ & $309.44 \pm 9.26 \mathrm{ab}$ & $311.35 \pm 23.94 a b$ & $298.00 \pm 2.97 b$ & $310.37 \pm 10.38 \mathrm{ab}$ & $322.73 \pm 3.57 a b$ \\
\hline Bitter & & $2426.00 \pm 104.38 a b$ & $2205.18 \pm 58.15 c$ & $2313.44 \pm 58.82 b$ & $2127.87 \pm 31.70 c$ & $2295.86 \pm 74.53 b$ & $2515.34 \pm 25.13 a$ \\
\hline Umami & & $755.22 \pm 43.99 \mathrm{ab}$ & $715.69 \pm 33.85 b c$ & $816.12 \pm 77.51 a$ & $670.11 \pm 34.67 c$ & $794.89 \pm 5.25 a b$ & $717.68 \pm 19.69 \mathrm{bc}$ \\
\hline Sweet & & $1802.72 \pm 41.28 c$ & $2048.50 \pm 239.37 b$ & $1217.77 \pm 54.81 d$ & $2197.73 \pm 42.71 b$ & $2427.28 \pm 21.68 a$ & $2438.30 \pm 15.39 a$ \\
\hline Total & & $5296.00 \pm 171.61 b$ & $5254.73 \pm 321.37 b$ & $4657.29 \pm 115.05 d$ & $5283.16 \pm 51.40 b$ & $5803.72 \pm 102.00 a$ & $5966.30 \pm 23.43 a$ \\
\hline
\end{tabular}

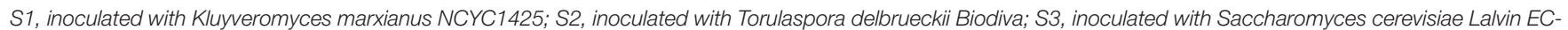

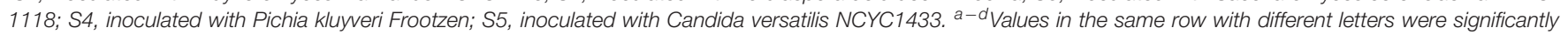
different $(P<0.05)$. Results: Mean $\pm S D, n=3$. 
TABLE 2 | Volatile compounds in fish sauce fermented with different yeasts $(\mu \mathrm{g} / \mathrm{L})$.

\begin{tabular}{|c|c|c|c|c|c|c|c|c|c|c|}
\hline \multirow[t]{2}{*}{ RT } & \multirow[t]{2}{*}{ KI } & \multirow[t]{2}{*}{ Compound name } & \multirow[t]{2}{*}{ Indentification } & \multirow[t]{2}{*}{ Fish hydrolysate } & \multicolumn{5}{|c|}{ Fish sauce (Day 7) } & \multirow[t]{2}{*}{ ОТ ( $\mu \mathrm{g} / \mathrm{L})$} \\
\hline & & & & & s1 & S2 & S3 & S4 & S5 & \\
\hline 6.08 & - & Ethyl acetate & MS & ND & $687.93 \pm 32.40 \mathrm{~b}$ & $31.17 \pm 2.69 c$ & $30.56 \pm 5.39 c$ & $3536.32 \pm 651.58 a$ & $2.75 \pm 0.37 c$ & $5^{a}$ \\
\hline 7.34 & 944 & Propanoic acid, ethyl ester & $\mathrm{MS}, \mathrm{KI}$ & ND & $7.90 \pm 1.47$ & ND & ND & ND & ND & $N F$ \\
\hline 8.55 & 1009 & Isobutyl acetate & $\mathrm{MS}, \mathrm{KI}$ & ND & ND & ND & ND & $22.60 \pm 9.83$ & ND & $25^{a}$ \\
\hline 10.05 & 1067 & Acetic acid, butyl ester & $\mathrm{MS}, \mathrm{KI}$ & ND & ND & ND & ND & $5.89 \pm 0.61$ & ND & $58^{\mathrm{a}}$ \\
\hline 11.30 & 1114 & 1-Butanol, 3- methyl-, acetate & $\mathrm{MS}, \mathrm{KI}$ & ND & $21.67 \pm 0.99 b$ & ND & $12.06 \pm 3.86 b$ & $1172.36 \pm 57.44 a$ & ND & $1.6^{\mathrm{a}}$ \\
\hline 12.67 & 1157 & Acetic acid, pentyl ester & $\mathrm{MS}, \mathrm{KI}$ & $N D$ & $4.80 \pm 0.24 b$ & ND & ND & $47.23 \pm 12.10 \mathrm{a}$ & ND & $43^{b}$ \\
\hline 15.30 & 1265 & Acetic acid, hexyl ester & $\mathrm{MS}, \mathrm{KI}$ & ND & $51.94 \pm 1.83 b$ & $0.46 \pm 0.12 \mathrm{c}$ & $22.24 \pm 4.36 c$ & $419.26 \pm 34.31 a$ & ND & $115^{\mathrm{C}}$ \\
\hline 16.51 & 1318 & n-Caproic acid vinyl ester & $\mathrm{MS}, \mathrm{KI}$ & $39.66 \pm 1.01 a$ & - & $1.96 \pm 0.19 b$ & $2.20 \pm 0.32 b$ & ND & ND & $N F$ \\
\hline 16.64 & 1325 & Heptanoic acid, ethyl ester & $\mathrm{MS}, \mathrm{KI}$ & ND & $1.02 \pm 0.08 c$ & $1.47 \pm 0.05 b$ & $2.38 \pm 0.41 a$ & ND & ND & $2^{\mathrm{a}}$ \\
\hline 17.20 & 1353 & Ethyl lactate & $\mathrm{MS}, \mathrm{KI}$ & ND & $18.87 \pm 0.56 \mathrm{ab}$ & $15.83 \pm 1.03 c$ & $17.71 \pm 2.44 \mathrm{bc}$ & ND & $20.91 \pm 1.47 a$ & NF \\
\hline 17.47 & 1366 & Acetic acid, heptyl ester & $\mathrm{MS}, \mathrm{KI}$ & ND & $9.26 \pm 0.92 b$ & ND & ND & $72.96 \pm 1.31 \mathrm{a}$ & ND & NF \\
\hline 17.63 & 1374 & Acetic acid, 2-ethylhexyl ester & $\mathrm{MS}, \mathrm{KI}$ & ND & ND & ND & ND & $7.52 \pm 2.50$ & ND & NF \\
\hline 18.27 & 1407 & 3-Hepten-1-ol, acetate & $\mathrm{MS}, \mathrm{KI}$ & ND & ND & ND & $0.58 \pm 0.08 b$ & $9.44 \pm 0.68 a$ & ND & NF \\
\hline 18.58 & 1427 & Octanoic acid, ethyl ester & $\mathrm{MS}, \mathrm{KI}$ & ND & $8.12 \pm 0.68 c$ & $14.09 \pm 1.63 b$ & ND & $19.17 \pm 3.44 a$ & ND & $19.4^{\mathrm{a}}$ \\
\hline 19.28 & 1469 & Acetic acid, octyl ester & MS,KI & ND & $6.11 \pm 0.60 \mathrm{bc}$ & ND & $11.01 \pm 1.14 b$ & $101.96 \pm 10.42 a$ & ND & $5000^{d}$ \\
\hline 19.84 & 1503 & 3-Octen-1-ol, acetate, (Z)- & $\mathrm{MS}, \mathrm{KI}$ & ND & ND & ND & ND & $5.27 \pm 0.45$ & ND & NF \\
\hline 19.95 & 1510 & 2,4-Hexadienoic acid, ethyl ester & $\mathrm{MS}, \mathrm{KI}$ & ND & $8.94 \pm 0.80 a$ & $3.48 \pm 0.19 b$ & $3.34 \pm 0.23 b$ & $3.06 \pm 0.22 b$ & ND & $N F$ \\
\hline 20.23 & 1530 & Non-anoic acid, ethyl ester & $\mathrm{MS}, \mathrm{KI}$ & ND & $10.55 \pm 1.55 a$ & $11.82 \pm 1.67 \mathrm{a}$ & $9.98 \pm 0.73 a$ & $10.37 \pm 0.54 a$ & ND & $377^{e}$ \\
\hline 20.85 & 1573 & Acetic acid, nonyl ester & $\mathrm{MS}, \mathrm{KI}$ & ND & $1.99 \pm 0.29 b$ & ND & $8.57 \pm 0.43 b$ & $70.70 \pm 8.74 a$ & ND & NF \\
\hline 20.97 & 1581 & 3-Methylbutyl 2-methoxyacetate & $\mathrm{MS}, \mathrm{KI}$ & ND & $7.13 \pm 0.77 a$ & ND & $2.19 \pm 0.45 b$ & ND & ND & NF \\
\hline 21.67 & 1634 & Decanoic acid, ethyl ester & $\mathrm{MS}, \mathrm{KI}$ & ND & $6.70 \pm 1.27 d$ & $28.39 \pm 8.09 b$ & $149.77 \pm 1.99 a$ & $18.13 \pm 2.80 c$ & ND & $5^{a}$ \\
\hline 21.96 & 1656 & Octanoic acid, 3-methylbutyl ester & $\mathrm{MS}, \mathrm{KI}$ & ND & ND & ND & $4.56 \pm 0.31$ & ND & ND & NF \\
\hline 22.02 & 1661 & 4-Decenoic acid, ethyl ester, (Z)- & $\mathrm{MS}, \mathrm{KI}$ & $N D$ & $1.49 \pm 0.28 a$ & $1.74 \pm 0.34 a$ & $1.40 \pm 0.21 a$ & $1.94 \pm 0.39 a$ & ND & NF \\
\hline 22.21 & 1676 & Acetic acid, decyl ester & $\mathrm{MS}, \mathrm{KI}$ & ND & ND & ND & $1.06 \pm 0.18 b$ & $5.43 \pm 0.57 a$ & ND & NF \\
\hline 22.36 & 1687 & Ethyl 9-decenoate & $\mathrm{MS}, \mathrm{KI}$ & ND & ND & $0.98 \pm 0.02 b$ & $4.49 \pm 0.25 a$ & ND & ND & NF \\
\hline 22.57 & 1704 & 3-Decen-1-ol, acetate, (Z)- & $\mathrm{MS}, \mathrm{KI}$ & ND & ND & ND & $3.10 \pm 0.21 b$ & $4.66 \pm 0.55 a$ & ND & NF \\
\hline 23.04 & 1744 & Acetic acid, phenylmethyl ester & $\mathrm{MS}, \mathrm{KI}$ & ND & ND & ND & ND & $4.53 \pm 1.18$ & ND & NF \\
\hline 24.08 & 1834 & Acetic acid, 2-phenylethyl ester & $\mathrm{MS}, \mathrm{KI}$ & $8.41 \pm 3.34 c$ & $253.50 \pm 21.41 b$ & $3.69 \pm 2.60 c$ & $4.50 \pm 0.51 \mathrm{c}$ & $1074.26 \pm 103.84 a$ & ND & $249.6^{a}$ \\
\hline 24.15 & 1841 & Dodecanoic acid, ethyl ester & $\mathrm{MS}, \mathrm{KI}$ & ND & $3.12 \pm 0.52 b$ & $2.60 \pm 0.66 b$ & $24.67 \pm 0.65 a$ & $3.62 \pm 0.88 b$ & ND & NF \\
\hline 24.82 & 1902 & $\begin{array}{l}\text { Propanoic acid, 2-phenylethyl } \\
\text { ester }\end{array}$ & $\mathrm{MS}, \mathrm{KI}$ & ND & $8.38 \pm 1.27$ & ND & ND & ND & ND & NF \\
\hline \multirow[t]{2}{*}{26.32} & 2049 & Tetradecanoic acid, ethyl ester & $\mathrm{MS}, \mathrm{KI}$ & ND & ND & ND & $1.50 \pm 0.11$ & ND & ND & NF \\
\hline & & Subtotal & & $48.07 \pm 3.43 \mathrm{e}$ & $1119.43 \pm 60.54 b$ & $119.31 \pm 9.21 d$ & $317.70 \pm 14.22 \mathrm{c}$ & $6620.74 \pm 814.00 a$ & $23.66 \pm 1.66 \mathrm{e}$ & \\
\hline 19.06 & 1455 & Acetic acid & $\mathrm{MS}, \mathrm{KI}$ & ND & $40.14 \pm 3.08 b$ & $16.06 \pm 2.40 \mathrm{c}$ & $22.95 \pm 9.07 c$ & $66.96 \pm 14.03 a$ & $25.48 \pm 1.48 \mathrm{c}$ & $5.5^{\mathrm{a}}$ \\
\hline 24.19 & 1844 & Hexanoic acid & MS,KI & $13.94 \pm 0.57 b$ & ND & $12.55 \pm 1.86 b$ & ND & $16.88 \pm 1.39 a$ & $13.97 \pm 0.84 b$ & $3000^{e}$ \\
\hline
\end{tabular}


RT KI Compound name Indentification Fish hydrolysate

Fish sauce (Day 7)

OT ( $\mu \mathrm{g} / \mathrm{L})$

\begin{tabular}{|c|c|c|c|c|c|c|c|c|c|c|}
\hline & & & & & s1 & S2 & s3 & S4 & S5 & \\
\hline 24.99 & 1918 & 4-Hexenoic acid & $\mathrm{MS}, \mathrm{KI}$ & ND & $10.71 \pm 0.46 a$ & $6.60 \pm 0.66 b$ & $5.67 \pm 0.91 b$ & ND & ND & NF \\
\hline 25.33 & 1951 & Heptanoic acid & $\mathrm{MS}, \mathrm{KI}$ & ND & ND & ND & $1.92 \pm 0.36 a$ & ND & $0.99 \pm 0.11 b$ & $3000^{e}$ \\
\hline 26.41 & 2058 & Octanoic acid & $\mathrm{MS}, \mathrm{KI}$ & $2.12 \pm 0.23 c$ & $2.97 \pm 0.24 c$ & $5.89 \pm 1.52 c$ & $50.53 \pm 2.62 a$ & $35.76 \pm 8.67 b$ & $1.52 \pm 0.63 c$ & $3000^{e}$ \\
\hline 27.39 & 2158 & Sorbic acid & $\mathrm{MS}, \mathrm{KI}$ & $14.30 \pm 1.21 a$ & $2.67 \pm 0.23$ & $3.35 \pm 0.82$ & $1.68 \pm 0.29$ & $11.42 \pm 2.57 b$ & $2.57 \pm 0.76 c$ & NF \\
\hline 27.45 & 2164 & Non-anoic acid & $\mathrm{MS}, \mathrm{KI}$ & $6.46 \pm 1.39 a$ & $5.87 \pm 0.51 \mathrm{ab}$ & $4.32 \pm 1.55 b c$ & $3.75 \pm 0.20 c$ & $7.53 \pm 0.82 a$ & $1.68 \pm 0.17 d$ & $3000^{e}$ \\
\hline 28.52 & 2270 & Decanoic acid & $\mathrm{MS}, \mathrm{KI}$ & ND & ND & $5.16 \pm 1.77 \mathrm{c}$ & $24.23 \pm 0.49 a$ & $7.55 \pm 1.45 b$ & $0.90 \pm 0.51 d$ & $10000^{e}$ \\
\hline \multirow[t]{2}{*}{31.11} & 2483 & Dodecanoic acid & $\mathrm{MS}, \mathrm{KI}$ & ND & ND & ND & $2.65 \pm 0.26$ & ND & ND & NF \\
\hline & & Subtotal & & $36.82 \pm 0.49 d$ & $62.36 \pm 3.18 c$ & $53.92 \pm 8.52 \mathrm{~cd}$ & $113.39 \pm 7.59 b$ & $146.10 \pm 28.28 a$ & $47.11 \pm 3.38 \mathrm{~cd}$ & \\
\hline 7.08 & 929 & Ethanol & $\mathrm{MS}, \mathrm{KI}$ & ND & $783.68 \pm 95.68 b$ & $724.63 \pm 55.38 b$ & $772.47 \pm 150.73 b$ & $1745.35 \pm 370.09 a$ & $506.51 \pm 59.38 b$ & $950000^{a}$ \\
\hline 14.26 & 1222 & 1-Butanol, 3-methyl- & $\mathrm{MS}, \mathrm{KI}$ & ND & $78.88 \pm 1.57 a$ & $46.54 \pm 3.58 b$ & ND & ND & ND & $4^{a}$ \\
\hline 17.57 & 1371 & 1-Hexanol & $\mathrm{MS}, \mathrm{KI}$ & ND & $64.39 \pm 3.75 b$ & $82.55 \pm 3.61 a$ & $74.58 \pm 0.96 a$ & ND & $35.77 \pm 2.04 \mathrm{c}$ & $5.7^{\mathrm{a}}$ \\
\hline 19.1 & 1458 & 1-Octen-3-ol & $\mathrm{MS}, \mathrm{KI}$ & $87.58 \pm 6.22$ & ND & ND & ND & ND & ND & $1.5^{\mathrm{a}}$ \\
\hline 19.46 & 1480 & 1-Heptanol & $\mathrm{MS}, \mathrm{KI}$ & ND & ND & $17.00 \pm 1.95$ & ND & ND & ND & $5.5^{\mathrm{a}}$ \\
\hline 20.05 & 1517 & 2-Ethyl-1-hexanol & $\mathrm{MS}, \mathrm{KI}$ & ND & ND & ND & ND & ND & $7.13 \pm 1.77$ & $\mathrm{NF}$ \\
\hline 22.8 & 1723 & 1-Non-anol & $\mathrm{MS}, \mathrm{KI}$ & ND & ND & $11.34 \pm 1.32$ & ND & ND & ND & $45.5^{\mathrm{a}}$ \\
\hline \multirow[t]{2}{*}{26.04} & 2020 & Phenylethyl alcohol & $\mathrm{MS}, \mathrm{KI}$ & ND & $136.87 \pm 8.85 \mathrm{a}$ & $74.05 \pm 11.49 b$ & $148.35 \pm 22.73 a$ & $134.29 \pm 26.71 a$ & $49.84 \pm 2.76 b$ & $564.2^{\mathrm{a}}$ \\
\hline & & Subtotal & & $87.58 \pm 6.22 d$ & $1088.23 \pm 103.16 b$ & $956.11 \pm 62.45 b$ & $995.40 \pm 176.12 b$ & $1879.64 \pm 391.06 a$ & $629.52 \pm 59.91 c$ & \\
\hline 7.81 & 971 & Pentanal & $\mathrm{MS}, \mathrm{KI}$ & $20.46 \pm 0.90 a$ & ND & ND & ND & ND & $3.38 \pm 0.29 b$ & $2501.3^{a}$ \\
\hline 10.35 & 1079 & Hexanal & $\mathrm{MS}, \mathrm{KI}$ & $209.11 \pm 10.01 a$ & ND & ND & ND & ND & $5.98 \pm 0.55 b$ & $5^{a}$ \\
\hline 11.79 & 1121 & 2-Pentenal, (E)- & $\mathrm{MS}, \mathrm{KI}$ & $9.02 \pm 1.40$ & ND & ND & ND & ND & ND & $N F$ \\
\hline 13.1 & 1175 & Heptanal & $\mathrm{MS}, \mathrm{KI}$ & $34.12 \pm 3.33$ & ND & ND & ND & ND & ND & $2.9^{a}$ \\
\hline 14.24 & 1221 & 2-Hexenal, (E)- & $\mathrm{MS}, \mathrm{KI}$ & $7.72 \pm 2.13$ & ND & ND & ND & ND & ND & $17^{e}$ \\
\hline 15.77 & 1284 & Octanal & $\mathrm{MS}, \mathrm{KI}$ & $30.05 \pm 1.90$ & ND & ND & ND & ND & ND & $0.6^{a}$ \\
\hline 16.69 & 1327 & 2-Heptenal, (Z)- & $\mathrm{MS}, \mathrm{KI}$ & $27.99 \pm 1.40$ & ND & ND & ND & ND & ND & $N F$ \\
\hline 16.87 & 1336 & 2-HexeNFI, 2-ethyl- & $\mathrm{MS}, \mathrm{KI}$ & $4.80 \pm 2.03$ & ND & ND & ND & ND & ND & $N F$ \\
\hline 17.96 & 1391 & Non-anal & $\mathrm{MS}, \mathrm{KI}$ & $118.83 \pm 16.83 a$ & $2.77 \pm 0.45 b$ & $3.10 \pm 0.81 b$ & $2.44 \pm 0.87 b$ & ND & $1.67 \pm 0.59 b$ & $1.1^{\mathrm{a}}$ \\
\hline 19.75 & 1497 & Decanal & $\mathrm{MS}, \mathrm{KI}$ & $4.47 \pm 1.34$ & ND & ND & ND & ND & ND & $0.1^{e}$ \\
\hline 19.87 & 1505 & 2,4-Heptadienal, (E,E)- & $\mathrm{MS}, \mathrm{KI}$ & $20.09 \pm 3.59$ & ND & ND & ND & ND & ND & $15.4^{\mathrm{a}}$ \\
\hline 20.38 & 1540 & 2-Non-enal, (E)- & $\mathrm{MS}, \mathrm{KI}$ & $19.82 \pm 3.52 \mathrm{a}$ & $1.39 \pm 0.76 b$ & $0.71 \pm 0.10 \mathrm{~b}$ & ND & ND & $0.40 \pm 0.10 b$ & $0.08^{\mathrm{e}}$ \\
\hline 20.41 & 1542 & Benzaldehyde & $\mathrm{MS}, \mathrm{KI}$ & ND & $8.06 \pm 2.70 d$ & $16.10 \pm 2.99 c$ & $24.47 \pm 4.10 b$ & $20.87 \pm 18.93 b$ & $42.95 \pm 4.75 a$ & $750.9^{a}$ \\
\hline 21.27 & 1602 & 2,4-Octadienal, (E,E)- & $\mathrm{MS}, \mathrm{KI}$ & $9.56 \pm 1.39$ & ND & ND & ND & ND & ND & NF \\
\hline 21.91 & 1652 & 2-Decenal, (E)- & $\mathrm{MS}, \mathrm{KI}$ & $19.09 \pm 5.00$ & ND & ND & ND & ND & ND & NF \\
\hline 22.08 & 1666 & Benzeneacetaldehyde & $\mathrm{MS}, \mathrm{KI}$ & $16.05 \pm 2.87 a$ & $1.54 \pm 0.09 b$ & ND & ND & ND & ND & $4^{\mathrm{e}}$ \\
\hline 22.72 & 1717 & 2,4-Non-adienal, (E,E)- & $\mathrm{MS}, \mathrm{KI}$ & $19.39 \pm 3.26$ & ND & ND & $N D$ & ND & ND & $0.09^{e}$ \\
\hline
\end{tabular}


TABLE 2 | Continued

\begin{tabular}{|c|c|c|c|c|c|c|c|c|c|c|}
\hline \multirow[t]{2}{*}{ RT } & \multirow[t]{2}{*}{ KI } & \multirow[t]{2}{*}{ Compound name } & \multirow[t]{2}{*}{ Indentification } & \multirow[t]{2}{*}{ Fish hydrolysate } & \multicolumn{5}{|c|}{ Fish sauce (Day 7) } & \multirow[t]{2}{*}{ OT $(\mu \mathrm{g} / \mathrm{L})$} \\
\hline & & & & & S1 & S2 & S3 & S4 & S5 & \\
\hline 22.92 & 1734 & Benzaldehyde, 3-ethyl- & $\mathrm{MS}, \mathrm{KI}$ & $3.40 \pm 0.62 a$ & ND & $0.17 \pm 0.03 b$ & ND & ND & ND & $\mathrm{NF}$ \\
\hline 23.24 & 1761 & 2-Undecenal & $\mathrm{MS}, \mathrm{KI}$ & $12.84 \pm 3.77 a$ & ND & $0.61 \pm 0.12 b$ & ND & ND & ND & NF \\
\hline 24.01 & 1828 & 2,4-Decadienal, (E,E)- & $\mathrm{MS}, \mathrm{KI}$ & $85.16 \pm 32.89 a$ & $2.52 \pm 0.71 b$ & $2.62 \pm 0.21 b$ & ND & ND & ND & $N F$ \\
\hline 24.25 & 1850 & Benzaldehyde, 2,5-dimethyl- & $\mathrm{MS}, \mathrm{KI}$ & $5.25 \pm 0.29 a$ & $2.03 \pm 0.16 c$ & $3.06 \pm 0.53 b$ & $1.98 \pm 0.19 \mathrm{c}$ & ND & $1.37 \pm 0.06 d$ & $N F$ \\
\hline \multirow[t]{2}{*}{27.22} & 2141 & Pentadecanal & $\mathrm{MS}, \mathrm{KI}$ & ND & $1.50 \pm 0.27 a$ & ND & $1.05 \pm 0.18 b$ & $1.78 \pm 0.35 a$ & $0.57 \pm 0.08 c$ & NF \\
\hline & & Subtotal & & $677.23 \pm 65.68 a$ & $19.82 \pm 3.58 c$ & $26.38 \pm 4.45 c$ & $29.93 \pm 3.83 c$ & $22.65 \pm 19.27 c$ & $52.93 \pm 5.99 b$ & \\
\hline 9.79 & 1057 & 2,3-Pentanedione & $\mathrm{MS}, \mathrm{KI}$ & $7.50 \pm 1.07$ & ND & ND & ND & ND & ND & $5505.6^{a}$ \\
\hline 13.71 & 1200 & 2-Heptanone, 4-methyl- & $\mathrm{MS}, \mathrm{KI}$ & ND & ND & $2.47 \pm 0.29 b$ & $2.28 \pm 0.27 b$ & ND & $3.12 \pm 0.26 a$ & $N F$ \\
\hline 14.87 & 1247 & 3-Octanone & $\mathrm{MS}, \mathrm{KI}$ & ND & ND & $2.05 \pm 0.23 b$ & ND & ND & $6.22 \pm 0.13 a$ & $21.5^{\mathrm{a}}$ \\
\hline 15.68 & 1281 & 2-Octanone & $\mathrm{MS}, \mathrm{KI}$ & ND & $3.10 \pm 0.53 b$ & $3.01 \pm 0.27 b$ & $3.21 \pm 0.61 b$ & ND & $5.77 \pm 0.43 a$ & $50.3^{a}$ \\
\hline 16.1 & 1298 & 1-Octen-3-one & $\mathrm{MS}, \mathrm{KI}$ & $12.18 \pm 2.15 a$ & $0.82 \pm 0.16 b$ & $1.30 \pm 0.09 b$ & $1.33 \pm 0.14 b$ & ND & ND & $N F$ \\
\hline 16.73 & 1329 & 6-Octen-2-one & $\mathrm{MS}, \mathrm{KI}$ & ND & ND & $1.70 \pm 0.02 b$ & $2.93 \pm 0.52 a$ & ND & $1.18 \pm 0.08 \mathrm{~b}$ & NF \\
\hline 18.3 & 1410 & 3-Octen-2-one & $\mathrm{MS}, \mathrm{KI}$ & $5.39 \pm 0.91$ & ND & ND & ND & ND & ND & NF \\
\hline 23.99 & 1826 & 1-Phenyl-2-butanone & $\mathrm{MS}, \mathrm{KI}$ & ND & $0.93 \pm 0.01 b$ & $0.89 \pm 0.10 \mathrm{~b}$ & $0.96 \pm 0.25 b$ & $1.79 \pm 0.27 a$ & $0.46 \pm 0.07 c$ & NF \\
\hline \multirow[t]{2}{*}{26.5} & 2067 & 2(3H)-Furanone, dihydro-5-pentyl- & $\mathrm{MS}, \mathrm{KI}$ & ND & $6.32 \pm 0.89 b$ & $5.58 \pm 0.97 b$ & $7.44 \pm 0.20 a$ & ND & $4.08 \pm 0.64 c$ & NF \\
\hline & & Subtotal & & $25.07 \pm 2.11 \mathrm{a}$ & $11.17 \pm 0.90 d$ & $16.99 \pm 0.49 c$ & $18.15 \pm 0.51 c$ & $1.79 \pm 0.27 \mathrm{e}$ & $21.95 \pm 1.14 b$ & \\
\hline 12.88 & 1166 & Dodecane & $\mathrm{MS}, \mathrm{KI}$ & ND & $7.34 \pm 1.31$ & ND & ND & ND & ND & NF \\
\hline 17.8 & 1383 & Tetradecane & MS,KI & ND & $4.82 \pm 0.94 b$ & $2.71 \pm 0.27 c$ & ND & $7.83 \pm 1.32 \mathrm{a}$ & ND & NF \\
\hline 18.78 & 1439 & Undecane, 3,8-dimethyl- & $\mathrm{MS}, \mathrm{KI}$ & ND & ND & ND & ND & $2.70 \pm 0.48$ & ND & NF \\
\hline \multirow[t]{2}{*}{19.61} & 1489 & Pentadecane & $\mathrm{MS}, \mathrm{KI}$ & $5.72 \pm 1.56 \mathrm{a}$ & ND & $4.19 \pm 0.95 b$ & ND & ND & ND & NF \\
\hline & & Subtotal & & $5.72 \pm 1.56 b$ & $12.15 \pm 2.24 a$ & $6.90 \pm 1.20 b$ & ND & $10.54 \pm 1.75 a$ & ND & \\
\hline 14.07 & 1214 & Furan, 2-pentyl- & MS,KI & $225.16 \pm 40.90 a$ & $58.94 \pm 8.96 b$ & $42.18 \pm 5.89 b c$ & $27.99 \pm 1.91 \mathrm{bc}$ & $40.81 \pm 1.49 \mathrm{bc}$ & $19.20 \pm 6.11 \mathrm{c}$ & $5.8^{\mathrm{a}}$ \\
\hline 15.95 & 1292 & Trans-2-(2-Pentenyl)furan & $\mathrm{MS}, \mathrm{KI}$ & $20.98 \pm 1.98 \mathrm{a}$ & $3.12 \pm 0.54 b$ & $2.50 \pm 0.32 \mathrm{bc}$ & $2.06 \pm 0.10 b c$ & ND & $1.32 \pm 0.32 c$ & $\mathrm{NF}$ \\
\hline \multirow[t]{2}{*}{20.21} & 1528 & 2-n-Octylfuran & $\mathrm{MS}, \mathrm{KI}$ & ND & $3.80 \pm 0.54 a$ & $2.54 \pm 0.61 b$ & ND & ND & ND & NF \\
\hline & & Subtotal & & $246.14 \pm 42.88 \mathrm{a}$ & $65.87 \pm 9.10 b$ & $47.22 \pm 6.78 \mathrm{bc}$ & $30.06 \pm 1.90 c$ & $40.81 \pm 1.49 \mathrm{bc}$ & $20.52 \pm 6.42 c$ & \\
\hline 18.41 & 1416 & Benzene, 1,3-bis(1,1-dimethylethyl)- & $\mathrm{MS}, \mathrm{KI}$ & ND & $73.88 \pm 5.93 b$ & $50.74 \pm 2.55 c$ & $59.47 \pm 10.58 \mathrm{bc}$ & $121.54 \pm 19.18 \mathrm{a}$ & $22.28 \pm 4.02 d$ & NF \\
\hline \multirow[t]{2}{*}{28.95} & 2310 & 2,4-Di-tert-butylphenol & $\mathrm{MS}, \mathrm{KI}$ & $21.38 \pm 3.09 c$ & $58.75 \pm 3.42 b$ & $46.56 \pm 6.67 b$ & $48.61 \pm 9.14 b$ & $122.22 \pm 16.23 a$ & $29.95 \pm 2.55 c$ & NF \\
\hline & & Subtotal & & $21.38 \pm 3.09 \mathrm{e}$ & $132.63 \pm 9.13 b$ & $97.30 \pm 7.40 \mathrm{c}$ & $108.08 \pm 15.76 b c$ & $243.76 \pm 33.14 a$ & $52.23 \pm 5.99 d$ & \\
\hline
\end{tabular}

KI, kovats index. S1, inoculated with Kluyveromyces marxianus NCYC1425; S2, inoculated with Torulaspora delbrueckii Biodiva; S3, inoculated with Saccharomyces cerevisiae Lalvin EC-1118; S4, inoculated with Pichia kluyveri Frootzen; S5, inoculated with Candida versatilis NCYC1433. All fish sauce fermented for 7 days. OT = Odor thresholds: a value obtained from Giri et al. (2010), b value obtained from Niu et al. (2019). c value obtained from Zhu et al. (2018), " value obtained from Zhao et al. (2017), " value obtained from Javed et al. (2018). ${ }^{a-e}$ Values in the same row with different letters were significantly different (P < 0.05). "ND": not detected. "NF": not found. Results: Mean $\pm S D, n=3$. 
$(P<0.05)$. In addition, there was no significant difference in ester concentrations between S5 and fish hydrolysate $(P>0.05)$. Furthermore, the largest amounts of ethyl acetate; 1-butanol, 3- methyl-, acetate and acetic acid, 2-phenylethyl ester over $1000 \mu \mathrm{g} / \mathrm{L}$ were also observed in S4.

Volatile acids including acetic acid and medium chain fatty acids were detected in all samples. The highest level of total acids was observed in S4 (147 $\mu \mathrm{g} / \mathrm{L})$, followed by S3 (114 $\mu \mathrm{g} / \mathrm{L})$, while no significant $(P>0.05)$ differences were observed between $\mathrm{S} 1$, S2, and S5 (48-63 $\mu \mathrm{g} / \mathrm{L})$. Several kinds of ketones were also detected after fermentation ranging from 2 to $22 \mu \mathrm{g} / \mathrm{L}$, compared to $26 \mu \mathrm{g} / \mathrm{L}$ of fish hydrolysate.

The total alcohols concentrations of S1-S4 were 1088, 956, 995, and $1880 \mu \mathrm{g} / \mathrm{L}$, respectively, significantly higher than S5 $(630 \mu \mathrm{g} / \mathrm{L})(P<0.05)$. A lower ethanol level was observed in S5 among the inoculated samples partially due to its lower glucose consumption (Table 1). The aldehydes mainly consisted of aliphatic aldehydes that decreased from 677 to $20-53 \mu \mathrm{g} / \mathrm{L}$ with yeast fermentation. Among them, significantly $(P<0.05)$ lower levels of straight chain aldehydes and unsaturated aldehydes were detected in S1-S4 $(2-8 \mu \mathrm{g} / \mathrm{L})$ than S5 $(12 \mu \mathrm{g} / \mathrm{L})$.

The fermentation with both soy sauce yeast and non-soy sauce yeast led to a significantly $(P<0.05)$ lower level of furans, especially 2-pentylfuran and trans-2-(2-pentenyl)furan. Furthermore, a significant increase of alkanes (C12-C15) was observed in $\mathrm{S} 1$ and $\mathrm{S} 4(P<0.05)$. However, it has been reported that C8-C19 alkanes did not have important effects on flavor because of their high odor threshold values (Xu et al., 2014).

\section{DISCUSSION}

\section{Yeast Populations and $\mathrm{pH}$ Changes During Fermentation}

This difference of yeast populations between S1-S4 and S5 was likely caused by the lack of salt in the fish hydrolysate. Fermentation with no salt led to a slower growth rate of C. versatilis NCYC 1433, which is a halotolerant strain isolated from fermenting soy sauce. The results indicated that these nonsoy sauce yeasts had good growth and survival in an unsalted and acidic environment relative to C. versatilis NCYC 1433. This initial $\mathrm{pH}$ decline was probably attributed to the release of organic acids (except added lactic acid) produced by yeasts (Figures 3C-G) and dissolved $\mathrm{CO}_{2}$.

\section{Utilization of Sugar and Production of Organic Acids}

The reduction of glucose content in all inoculated samples was due to the metabolism of yeasts. All non-soy sauce yeasts had a higher rate of glucose consumption than soy sauce yeast (S5), indicating that non-soy sauce yeasts had a higher fermentative activity in the unsalted system. Normally, glucose is firstly convert into pyruvic acid (Figure 3C) via glycolysis in the cytoplasmic matrix, with the production of citric acid, malic acid and succinic acid by the TCA cycle (Chen and Liu, 2016; Varela, 2016), or converted into ethanol and carbon dioxide via alcoholic fermentation (Lu et al., 2017b). Ethanol is not only an important flavor carrier but also a precursor to ethyl esters, which are important odorants in yeast fermentation (Table 2). It was also reported that high levels of residual sugars could cause a lagging effect on perception of aromas (Villamor et al., 2013). In addition, the heat treatment after 2\% (w/v) glucose addition might lead to the Maillard reaction between glucose and protein/peptides/amino acids, resulting in a lower level of glucose $(16.85 \mathrm{~g} / \mathrm{L})$.

The additional pyruvic acid in S4 was possibly derived from glycerol pyruvate pathway (Ciani et al., 2006). An opposite result was reported by Chen and Liu (2016), who found S. cerevisiae Lalvin EC-1118 produced higher amounts of pyruvic acid than $P$. kluyveri Frootzen in lychee wine. This phenomenon could be caused by the different fermentation conditions. Moreover, the reduced pyruvic acid of S4 in the late fermentation could be transformed into acetaldehyde (Cherry et al., 2012). Citric acid (Figure 3D), malic acid (Figure 3E) and succinic acid (Figure 3F) are known to be the critical intermediate products of the TCA cycle. The faster increasing rate of succinic acid observed in S3 was possibly attributed to glyoxylate cycle for succinic acid production in S. cerevisiae Lalvin EC-1118 strains in addition to the TCA cycle (Raab and Lang, 2011; Yan et al., 2014). Acetic acid is believed to be produced in yeast by the enzymatic oxidation of acetaldehyde, which is the product of pyruvic acid decarboxylation (Freer, 2002).

\section{Changes of Free Amino Acids}

The interaction between these taste-active amino acids can make a direct contribution to the taste of fish sauce. Free amino acids are not only linked to the taste, but also important aroma precursors. It is reported that valine, leucine and phenylalanine can be catabolized to generate 2-methyl-1-propanol (isobutyl alcohol), 3-methyl-1-butanol (isoamyl alcohol) and phenylethyl alcohol, respectively, via the Ehrlich pathway (Chen and Liu, 2016). These alcohols can be further converted into volatile esters to affect the overall aroma profile by yeasts via esterification or alcoholysis (Gao et al., 2018).

3-Methyl-1-butanol, the metabolic product of leucine, was detected in fish sauce, which is subsequently converted into 1butanol, 3- methyl-, acetate by yeast (Gao et al., 2018). The leucine concentrations of samples fermented with non-soy sauce yeasts (S1 and S3) were significantly lower than that of soy sauce yeast $(P<0.05)$, with detection of more 3-methyl-1-butanol or 3-methyl-1-butanol, acetate (Table 2). And no 3-methyl-1butanol and its corresponding ester was found in S5. The results indicated the non-soy sauce yeasts could utilize leucine for aroma substances production.

The valine concentrations in S1, S3, and S4 significantly decreased after fermentation $(P<0.05)$. Similarly, the declined valine might be converted into isobutyl alcohol or its corresponding ester (isobutyl acetate). Actually, isobutyl acetate was only found in S4 and no isobutyl alcohol was detected at the end of fermentation (Table 2). Moreover, phenylethyl alcohol and its related esters (acetic acid, 2-phenylethyl ester and propanoic acid, 2-phenylethyl ester; Table 2) were detected in all samples except S5. The results suggested that the non-soy 
sauce yeasts could also utilize valine and phenylalanine to generate odorants.

Methionine has been reported as a precursor for distinct odorants of fish sauce such as dimethyl sulfide, dimethyl disulfide and dimethyl trisulfide (Udomsil et al., 2011). However, no sulfur-containing compounds were detected in all samples (Table 2), indicating that these non-soy sauce yeasts could not utilize methionine to produce volatile sulfurcontaining compounds in the unsalted and acidic system during a short period.

\section{Generation of Volatile Compounds}

For starting matrix, the aldehydes consisted mostly of straight chain aldehydes and unsaturated aldehydes, which were the metabolites of unsaturated fatty acid, caused by fish endogenous enzymes or autoxidation, especially in heat treatment. Furans could be formed by the Amadori rearrangement pathways (Giri et al., 2010), which was associated with heat treatment.

A higher level of esters in S4 and S1 indicated that P. kluyveri Frootzen and K. marxianus NCYC1425 had a stronger esterproducing capacity in fish hydrolysate fermentation. More acetate esters and ethyl esters in S4 could be attributed to higher levels of precursors such as acetic acid and ethanol generated by P. kluyveri Frootzen, which agree with Lu et al. (2017b). Ester compounds are common volatile components of flavor in food, providing the desirable fruity aroma to the overall aroma profile, due to their low odor thresholds (Zhao et al., 2017; Javed et al., 2018; Zhu et al., 2018; Niu et al., 2019). In this study, ethyl acetate; 1-butanol, 3- methyl-, acetate; decanoic acid, ethyl ester; and acetic acid, pentyl ester (S4); acetic acid, hexyl ester (S4); heptanoic acid, ethyl ester (S3); acetic acid, 2-phenylethyl ester (S4) could contribute to the overall aroma as their concentrations exceed their odor thresholds.

S. cerevisiae was reported to have the lipolytical ability to release fatty acids in the previous study (Gao et al., 2016b). It was considered that volatile straight chain fatty acids and ketones were responsible for the cheesy odor of fish sauce (Peralta et al., 1996; Fukami et al., 2002). Meanwhile, S4 and S3 had a significant higher level of fatty acids than other samples $(P<0.05)$. Therefore, P. kluyveri Frootzen and S. cerevisiae Lalvin EC-1118 had a higher potential to enhance the cheesy odor of fish sauce.

One endogenous alcohol (1-octen-3-ol) was detected in fish hydrolysate, which could be generated from the oxidation of linoleic acid or arachidonic acid (Zhao et al., 2015). A new

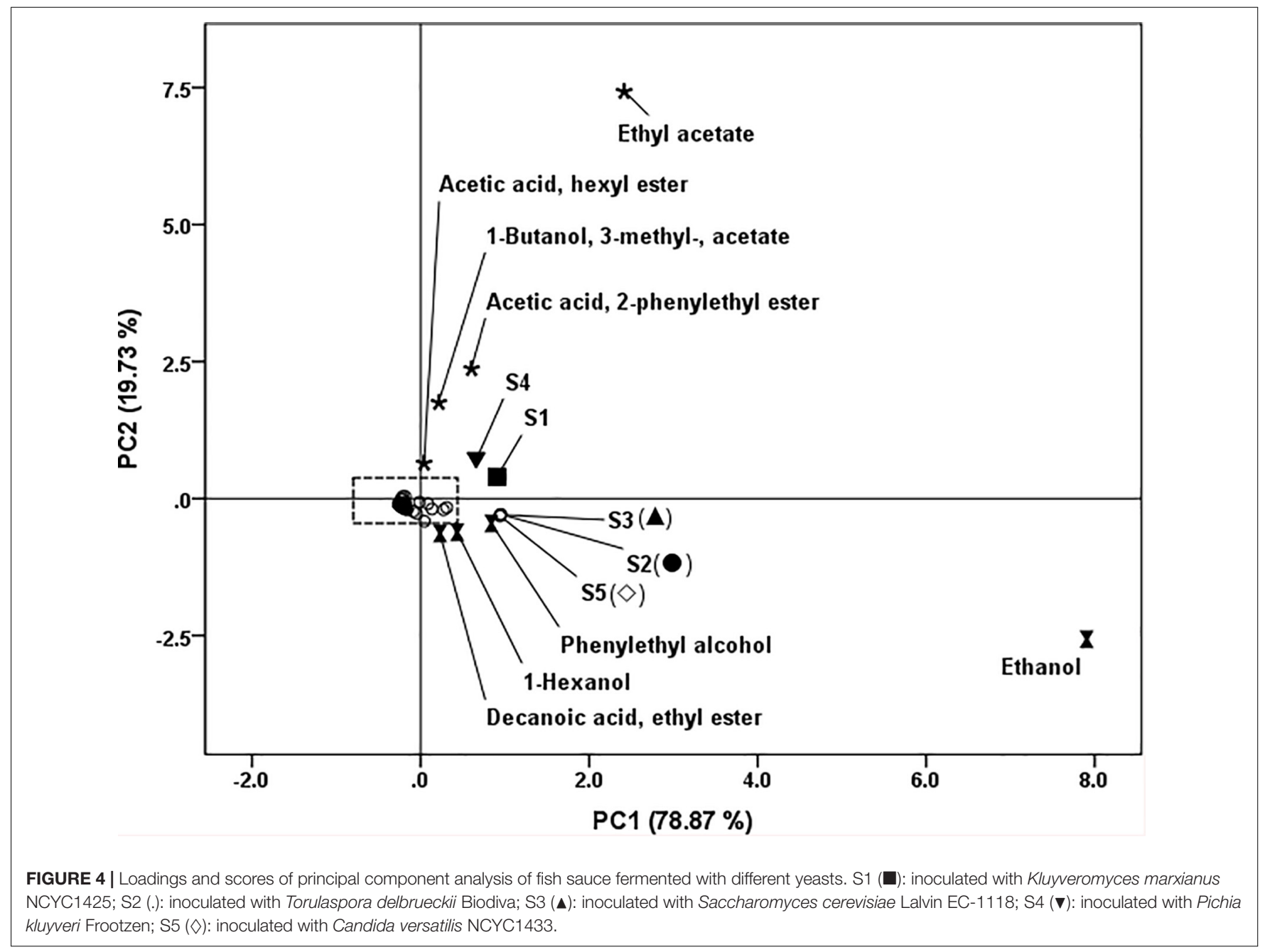


series of aliphatic alcohols (C2-C9) and phenylethyl alcohol were detected after fermentation. These higher alcohols are produced either from sugars or from amino acids (via Ehrlich mechanism) (Chua et al., 2018). Although present at higher levels, ethanol made a less direct contribution to flavor, due to its relatively high odor threshold (Peralta et al., 1996; Giri et al., 2010). However, it could affect the overall aroma as the precursor of esters as well as aroma compound carrier.

This aldehydes decrease was probably caused by its degradation, reduction to alcohols or further reactions with other compounds, such as amino acids and Maillard other hand, the aerobic respiration of yeast consumed dissolved oxygen and thus inhibited the autoxidation to reduce the production of aldehydes. Normally, straight chain aldehydes generally provide green and grassy aromas, while unsaturated aldehydes are linked with fishy note (Giri et al., 2010). Moreover, significantly $(P<0.05)$ lower levels of straight chain aldehydes and unsaturated aldehydes were detected in S1-S4, indicating wine and dairy yeasts, especially P. kluyveri Frootzen, exhibited a variously stronger ability to diminish some certain aldehydes related to distinct odor of fish sauce.

It has been reported that unsaturated aldehydes, pyridines, sulfur-containing compounds and amines contributed cooperatively to the undesirable odor of fish sauce. The volatile compounds detected in this study contained esters, acids, alcohols, aldehydes, ketones, alkanes, furans and aromatics, but not the above-mentioned fish sauce distinct odorants. In addition, different degrees of yeasty aroma could be smelt from the samples, depending on yeast. These findings indicated that the fermentation of non-soy sauce yeasts could effectively diminish the unpleasant smell of fishy, sweaty, rancid and fecal notes, and enrich the favorable flavors at the same time.

\section{Principal Component Analysis}

In order to evaluate the aroma profile of fish sauce with different yeasts treatments, principal component analysis (PCA) was conducted using the data of volatiles from Table 2 except fish hydrolysate volatiles. The results are shown in Figure 4. Two principal components were extracted from five variables accounted for $98.60 \%$ (PC1 78.87\%, PC2 19.73\%). The samples were classified into two separate groups. The first group contained S1 and S4; the second group contained S2, S3, and S5. S1 and S4, located at the first quadrant, were correlated with acetate esters (ethyl acetate; 1-butanol, 3- methyl-, acetate; acetic acid, hexyl ester; acetic acid, 2-phenylethyl ester). S2, S3 and

\section{REFERENCES}

Chen, D., and Liu, S.-Q. (2016). Impact of simultaneous and sequential fermentation with Torulaspora delbrueckii and Saccharomyces cerevisiae on non-volatiles and volatiles of lychee wines. LWT-Food Sci. Technol. 65, 53-61. doi: 10.1016/j.lwt.2015.07.050

Cherry, J. M., Hong, E. L., Amundsen, C., Balakrishnan, R., Binkley, G., Chan, E. T., et al. (2012). Saccharomyces genome database: the genomics resource of budding yeast. Nucleic Acids Res. 40, D700-D705. doi: 10.1093/nar/gkr 1029
S5, located at the fourth quadrant, were related to ethanol, 1hexanol, 2-phenylethyl alcohol and decanoic acid, ethyl ester. The distribution of volatile compounds in the quadrants provided the information about the differences of wine and dairy yeast.

\section{CONCLUSION}

This research demonstrated that $T$. delbrueckii Biodiva, S. cerevisiae Lalvin EC-1118, P. kluyveri Frootzen and K. marxianus NCYC1425 had a higher fermentative activity than C. versatilis NCYC1433 (a soy sauce yeast) in a fish sauce production from tilapia fish head hydrolysate with salt-free and acidic conditions in a short period. Especially, inoculation of K. marxianus NCYC1425 and P. kluyveri Frootzen could effectively diminish the distinctive notes of traditional fish sauce, and enrich the fruity note at the same time. It concluded that nonsoy sauce yeasts with a high aromatic capacity are suitable for fish sauce flavor modification and to develop a fast fermentation process for salt-free fish sauce from fish head. These applications could increase the acceptability of fish sauce and improve the utilization of fish by-products.

\section{DATA AVAILABILITY STATEMENT}

All datasets generated for this study are included in the manuscript/supplementary files.

\section{AUTHOR CONTRIBUTIONS}

SL and PG designed the study. SL and WX supervised the study. PG and XL performed the experiments. PG wrote the manuscript. SL reviewed the manuscript.

\section{FUNDING}

This research was partially funded by the National Natural Science Foundation of China (31801575), the Earmarked Fund for China's Agricultural Research System (CARS-45), Postdoctoral Science Foundation of China (2018M642167), Collaborative Innovation Center of Food Safety and Quality Control in Jiangsu Province, China and 2014GB2C100297, and National First-class Discipline Program of Food Science and Technology (JUFSTR20180201).

Chua, J. Y., Lu, Y., and Liu, S. Q. (2018). Evaluation of five commercial nonSaccharomyces yeasts in fermentation of soy (tofu) whey into an alcoholic beverage. Food Microbiol. 76, 533-542. doi: 10.1016/j.fm.2018.07.016

Ciani, M., Beco, L., and Comitini, F. (2006). Fermentation behaviour and metabolic interactions of multistarter wine yeast fermentations. Int. J. Food Microbiol. 108, 239-245. doi: 10.1016/j.ijfoodmicro.2005.11.012

Contreras, A., Hidalgo, C., Henschke, P. A., Chambers, P. J., Curtin, C., and Varela, C. (2014). Evaluation of non-Saccharomyces yeasts for the reduction of alcohol content in wine. Appl. Environ. Microbiol. 80, 1670-1678. doi: 10.1128/aem. 03780-13 
Devanthi, P. V. P., Linforth, R., Onyeaka, H., and Gkatzionis, K. (2018). Effects of co-inoculation and sequential inoculation of Tetragenococcus halophilus and Zygosaccharomyces rouxii on soy sauce fermentation. Food Chem. 240, 1-8. doi: 10.1016/j.foodchem.2017.07.094

FAO (2018). Food and Agricultural Organization Yearbook. Rome: Fishery Statistics.

Freer, S. N. (2002). Acetic acid production by Dekkera/Brettanomyces yeasts. World J. Microbiol. Biotechnol. 18, 271-275. doi: 10.1023/A:1014927129259

Fukami, K., Funatsu, Y., Kwasaki, K., and Watabe, S. (2004). Improvement of fish-sauce odor by treatment with bacteria isolated from the fish-sauce mush (Moromi) made from frigate mackerel. J. Food Sci. 69, M45-M49.

Fukami, K., Ishiyama, S., Yaguramaki, H., Masuzawa, T., Nabeta, Y., Endo, K., et al. (2002). Identification of distinctive volatile compounds in fish sauce. J. Agric. Food Chem. 50, 5412-5416. doi: 10.1021/jf020405y

Gao, P., Jiang, Q., Xu, Y., and Xia, W. (2018). Biosynthesis of acetate esters by dominate strains, isolated from chinese traditional fermented fish (Suan yu). Food Chem. 244, 44-49. doi: 10.1016/j.foodchem.2017.10.007

Gao, P., Wang, W., Jiang, Q., Xu, Y., and Xia, W. (2016a). Effect of autochthonous starter cultures on the volatile flavour compounds of Chinese traditional fermented fish (Suan yu). Int. J. Food Sci. Technol. 51, 1630-1637. doi: 10.1111/ ijfs. 13134

Gao, P., Wang, W., Xia, W., Xu, Y., and Jiang, Q. (2016b). Lipolysis and lipid oxidation caused by Staphylococcus xylosus 135 and Saccharomyces cerevisiae 31 isolated from Suan yu, a traditional Chinese low-salt fermented fish. Int. J. Food Sci. Technol. 51, 419-426. doi: 10.1111/ijfs.12997

Giri, A., Osako, K., Okamoto, A., and Ohshima, T. (2010). Olfactometric characterization of aroma active compounds in fermented fish paste in comparison with fish sauce, fermented soy paste and sauce products. Food Res. Int. 43, 1027-1040. doi: 10.1016/j.foodres.2010.01.012

Harada, R., Yuzuki, M., Ito, K., Shiga, K., Bamba, T., and Fukusaki, E. (2017). Influence of yeast and lactic acid bacterium on the constituent profile of soy sauce during fermentation. J. Biosci. Bioeng. 123, 203-208. doi: 10.1016/j.jbiosc. 2016.08.010

Javed, H. U., Wang, D., Shi, Y., Wu, G. F., Xie, H., Pan, Y. Q., et al. (2018). Changes of free-form volatile compounds in pre-treated raisins with different packaging materials during storage. Food Res. Int. 107, 649-659. doi: 10.1016/j.foodres. 2018.03.019

Jiang, J.-J., Zeng, Q.-X., and Zhu, Z.-W. (2008). Analysis of volatile compounds in traditional chinese fish sauce (Yu Lu). Food Bioprocess. Technol. 4, 266-271. doi: 10.1007/s11947-008-0173-8

Kristinsson, H. G., and Rasco, B. A. (2000). Fish protein hydrolysates: production, biochemical, and functional properties. Crit. Rev. Food Sci. 40, 43-81. doi: 10.1080/10408690091189266

Lopetcharat, K., Choi, Y. J., Park, J. W., and Daeschel, M. A. (2001). Fish sauce products and manufacturing: a review. Food Rev. Int. 17, 65-88. doi: 10.1081/ fri- 100000515

Lu, Y., Chan, L.-J., Li, X., and Liu, S.-Q. (2018). Effects of different inoculation strategies of Saccharomyces cerevisiae and Williopsis saturnus on chemical components of mango wine. LWT-Food Sci. Technol. 87, 85-92. doi: 10.1016/ j.lwt.2017.08.074

Lu, Y., Chua, J.-Y., Huang, D., Lee, P.-R., and Liu, S.-Q. (2017a). Chemical consequences of three commercial strains of Oenococcus oeni co-inoculated with Torulaspora delbrueckii in durian wine fermentation. Food Chem. 215, 209-218. doi: 10.1016/j.foodchem.2016.07.158

Lu, Y., Voon, M. K. W., Chua, J.-Y., Huang, D., Lee, P.-R., and Liu, S.-Q. (2017b). The effects of co- and sequential inoculation of Torulaspora delbrueckii and Pichia kluyveri on chemical compositions of durian wine. Appl. Microbiol. Biotechnol. 101, 7853-7863. doi: 10.1007/s00253-017-8527-7

Niu, Y., Wang, R., Xiao, Z., Zhu, J., Sun, X., and Wang, P. (2019). Characterization of ester odorants of apple juice by gas chromatographyolfactometry, quantitative measurements, odour threshold, aroma intensity and electronic nose. Food Res. Int. 120, 92-101. doi: 10.1016/j.foodres.2019. 01.064
Peralta, R. R., Shimoda, M., and Osajima, Y. (1996). Further identification of volatile compounds in fish sauce. J. Agric. Food Chem. 44, 3606-3610. doi: $10.1021 /$ jf9603036

Raab, A. M., and Lang, C. (2011). Oxidative versus reductive succinic acid production in the yeast Saccharomyces cerevisiae. Bioeng. Bugs 2, 120-123. doi: 10.4161/bbug.2.2.14549

Robert, M., Zatylny-Gaudin, C., Fournier, V., Corre, E., Le Corguillé, G., Bernay, B., et al. (2015). Molecular characterization of peptide fractions of a tilapia (Oreochromis niloticus) by-product hydrolysate and in vitro evaluation of antibacterial activity. Process. Biochem. 50, 487-492. doi: 10.1016/j.procbio. 2014.12.022

Singracha, P., Niamsiri, N., Visessanguan, W., Lertsiri, S., and Assavanig, A. (2017). Application of lactic acid bacteria and yeasts as starter cultures for reducedsalt soy sauce (moromi) fermentation. LWT-Food Sci. Technol. 78, 181-188. doi: 10.1016/j.lwt.2016.12.019

Udomsil, N., Rodtong, S., Choi, Y. J., Hua, Y., and Yongsawatdigul, J. (2011). Use of Tetragenococcus halophilus as a starter culture for flavor improvement in fish sauce fermentation. J. Agric. Food Chem. 59, 8401-8408. doi: 10.1021/jf201953v

Varela, C. (2016). The impact of non-Saccharomyces yeasts in the production of alcoholic beverages. Appl. Microbiol. Biotechnol. 100, 9861-9874. doi: 10.1007/ s00253-016-7941-6

Villamor, R. R., Evans, M. A., Mattinson, D. S., and Ross, C. F. (2013). Effects of ethanol, tannin and fructose on the headspace concentration and potential sensory significance of odorants in a model wine. Food Res. Int. 50, 38-45. doi: 10.1016/j.foodres.2012.09.037

Xu, Y., Liu, Y., Jiang, C., Zhang, C., Li, X., Zhu, D., et al. (2014). Determination of volatile compounds in turbot (Psetta maxima) during refrigerated storage by headspace solid-phase microextraction and gas chromatographymass spectrometry. J. Sci. Food Agric. 94, 2464-2471. doi: 10.1002/jsfa. 6581

Yan, D., Wang, C., Zhou, J., Liu, Y., Yang, M., and Xing, J. (2014). Construction of reductive pathway in Saccharomyces cerevisiae for effective succinic acid fermentation at low $\mathrm{pH}$ value. Bioresour. Technol. 156, 232-239. doi: 10.1016/j. biortech.2014.01.053

Yoshikawa, S., Kurihara, H., Kawai, Y., Yamazaki, K., Tanaka, A., Nishikiori, T., et al. (2010). Effect of halotolerant starter microorganisms on chemical characteristics of fermented chum salmon (Oncorhynchus keta) sauce. J. Agric. Food Chem. 58, 6410-6417. doi: 10.1021/jf904548u

Zhao, P., Qian, Y., He, F., Li, H., and Qian, M. (2017). Comparative characterization of aroma compounds in merlot wine by lichrolut-en-based aroma extract dilution analysis and odor activity value. Chem. Percept. 10, 149-160. doi: 10.1007/s12078-017-9236-4

Zhao, Q., Shen, Q., Guo, R., Wu, J., and Dai, Z.-Y. (2015). Characterization of flavor properties from fish (Collichthys niveatus) through enzymatic hydrolysis and the maillard reaction. J. Aquat. Food Prod. Technol. 25, 482-495. doi: 10.1080/10498850.2013.873965

Zhu, J., Wang, L., Xiao, Z., and Niu, Y. (2018). Characterization of the key aroma compounds in mulberry fruits by application of gas chromatography-olfactometry (GC-O), odor activity value (OAV), gas chromatography-mass spectrometry (GC-MS) and flame photometric detection (FPD). Food Chem. 245, 775-785. doi: 10.1016/j.foodchem.2017. 11.112

Conflict of Interest: The authors declare that the research was conducted in the absence of any commercial or financial relationships that could be construed as a potential conflict of interest.

Copyright (c) $2019 \mathrm{Gao}, \mathrm{Xia}, \mathrm{Li}$ and Liu. This is an open-access article distributed under the terms of the Creative Commons Attribution License (CC BY). The use, distribution or reproduction in other forums is permitted, provided the original author(s) and the copyright owner(s) are credited and that the original publication in this journal is cited, in accordance with accepted academic practice. No use, distribution or reproduction is permitted which does not comply with these terms. 